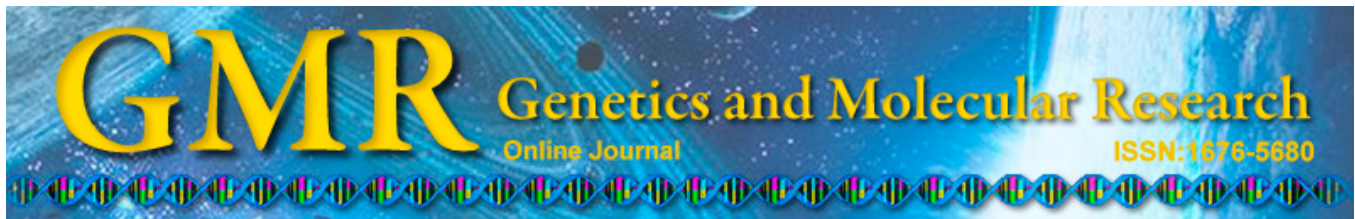

\title{
FlgN plays important roles in the adhesion of Aeromonas hydrophila to host mucus
}

\author{
X. Jiang1,2, Y.X. Qin ${ }^{1,2}$, G.F. Lin ${ }^{1,2}$, L. Huang ${ }^{1,2}$, B. Huang ${ }^{1,2}$, W.S. Huang ${ }^{1,2}$ \\ and Q.P. Yan ${ }^{1,2}$ \\ ${ }^{1}$ Fisheries College, Jimei University, Xiamen, Fujian, China \\ ${ }^{2}$ Key Laboratory of Healthy Mariculture for the East China Sea, \\ Ministry of Agriculture, Jimei University, Xiamen, Fujian, China \\ Corresponding author: Q.P. Yan \\ E-mail: yanqp@jmu.edu.cn
}

Genet. Mol. Res. 14 (2): 6376-6386 (2015)

Received November 4, 2014

Accepted February 5, 2015

Published June 11, 2015

DOI http://dx.doi.org/10.4238/2015.June.11.13

\begin{abstract}
Adhesion to the host mucus is a crucial step in the early infection stage of pathogenic bacteria. To investigate the mechanisms of the adhesion of Aeromonas hydrophila to its host mucus, a mutant library was constructed using the mini-Tn 10 transposon mutagenesis system. Of 276 individual colonies, the mutant strain with the most attenuated adhesion ability in this study was screened out and designated A77. Molecular analysis showed that a 414-bp sequence flanking mini-Tn10 in A77 had the highest identity (97\%) with the bacterial flagellar protein gene $f g N$. A complemented strain $f g N+$ was constructed and the biological characteristics of the wild-type, mutant A77, and complemented $f g N+$ strains were investigated. The results showed that the decreased abilities of motility, adhesion to mucus, and biofilm formation in the mutant strain were partially recovered in the complemented $\operatorname{fg} N+$ strain, which suggested that $\operatorname{fg} N$ plays an important role in the adhesion of $A$. hydrophila to its host.
\end{abstract}

Key words: Adhesion; Aeromonas hydrophila; $\operatorname{fgN}$; Mini-Tn10Km; ELISA 


\section{INTRODUCTION}

Pathogen adhesion to the cells and mucosal surfaces of the host is a critical step in the early infection process (Acord et al., 2005). For many pathogenic bacteria, it is only when they adhere to sensitive cells through adhesins they produce toxins in the focus of infection and eventually penetrate the tissues of the host. For this reason, bacterial adhesion has been regarded as a virulence factor of pathogenic bacteria (Chhatwal, 2002). In recent decades, the mechanisms of adhesion of many clinical pathogens to their hosts have been elucidated. Studies on Burkholderia pseudomallei demonstrated that the pilA deletion mutant strain JAB16 showed lower adhesion to human epithelial cell lines than the wild type strain $B$. pseudomallei K96243, and that Caenorhabditis elegans infected with JAB16 survived significantly longer than those infected with K96243 (Essex-Lopresti et al., 2005). Kelly et al. (2004) studied the adhesion of Candida albicans and found that a CAACE2 gene knock-out caused a two-fold decrease in adhesion to 96-well polystyrene plates, and that female mice infected with the wild type strain survived only for 5-7 days, while those infected with the CaACE2 gene knock-out mutant strain remained alive after 28 days.

Although adhesion is an important virulence factor for pathogens, most studies on bacterial adhesion have focused on clinical pathogenic bacteria; hardly any fish pathogens have been studied to uncover the mechanisms of their adhesion.

Aeromonas hydrophila is a widespread representative of Aeromonas and is found in water, water inhabitants, and in domestic animals and food. It is not only responsible for several kinds of diseases of farmed and feral fish including hemorrhagic ulceration and hemorrhagic septicemia, but is also responsible for gastrointestinal and extra-intestinal infections of humans; for example, acute diarrheal disease, bacteremia, and meningitis, among others (Daskalov, 2006). Several structures or molecules have previously been demonstrated to be related to the pathogenicity of $A$. hydrophila, such as toxins, proteases, outer membrane proteins, lipopolysaccharide, and motility proteins, among others (Merino et al., 1996; Gavín et al., 2003; Khajanchi et al., 2009; McCoy et al., 2010; Khushiramani et al., 2012). However, the adhesion of $A$. hydrophila is not well understood. Pianetti et al. (2012) once evaluated the adhesion properties of a A. hydrophila strains isolated from fish following prolonged storage at refrigerated and room temperatures in the presence of different $\mathrm{NaCl}$ concentrations, and using microscopic analysis, determined that adhesive capacity decreased over time with an increase $\mathrm{NaCl}$ concentrations. However, there have been no reports on the mechanisms of adhesion of $A$. hydrophila. In this study, we explore the role of genes related to A. hydrophila adhesion through construction of mutant and complemented strains, which will contribute to a better understanding of the adhesion mechanisms of $A$. hydrophila.

\section{MATERIAL AND METHODS}

\section{Bacterial strains, plasmids, and growth conditions}

Strains and plasmids used in this study are listed in Table 1. A. hydrophila strains were routinely grown overnight in Luria-Bertani (LB) broth at $28^{\circ} \mathrm{C}$, unless otherwise indicated. Escherichia coli strains were grown in LB at $37^{\circ} \mathrm{C}$. The semi-soft medium used for motility assays was LB plus $0.3 \%$ agar. The medium was supplemented with the appropriate antibiotics 
at the following concentrations when required: $100 \mu \mathrm{g} / \mathrm{mL}$ kanamycin $(\mathrm{Km}) ; 50 \mu \mathrm{g} / \mathrm{mL}$ ampicillin and $50 \mu \mathrm{g} / \mathrm{mL}$ streptomycin (Sm); or $25 \mu \mathrm{g} / \mathrm{mL}$ chloromycetin.

\begin{tabular}{|c|c|c|}
\hline Strains or plasmids & Characteristic(s) & Source or reference \\
\hline \multicolumn{3}{|l|}{ Strains } \\
\hline \multicolumn{3}{|l|}{ A. hydrophila } \\
\hline W & Wild-type strain $\left(\mathrm{Sm}^{\mathrm{R}}\right)$, isolated from diseased eel & This study \\
\hline A77 & Mini-Tn $10 \mathrm{Km}$ insertion mutant deficient in adhesion $\left(\mathrm{Sm}^{\mathrm{R}} \mathrm{Km}^{\mathrm{R}}\right)$ & This study \\
\hline flgN + & A77 complementation with intact $f g N$ gene $\left(\mathrm{Km}^{\mathrm{R}} \mathrm{Cm}^{\mathrm{R}}\right)$ & This study \\
\hline \multicolumn{3}{|c|}{ 月1 } \\
\hline $\operatorname{Sm} 10$ & thi thr leu tonA lacY supE recA RP4-2-Tc::Mu::Km ( $\lambda$ pir) & Herrero et al. (1990) \\
\hline $\mathrm{DH}_{5} \alpha$ & F-endA hdsR17( $\left.\mathrm{r}_{\mathrm{K}}^{-} \mathrm{m}_{\mathrm{K}}^{+}\right)$supE44 thi-l recAl gyr-A96 80lacZ & Hanahan (1983) \\
\hline \multicolumn{3}{|c|}{ - } \\
\hline pLOFKm & $\mathrm{Ap}^{\mathrm{R}} ; \mathrm{Tn} 10$-based delivery plasmid with $\mathrm{Km}^{\mathrm{R}}$ & Herrero et al. (1990) \\
\hline pACYC184 & Cloning vector, $\mathrm{Tc}^{\mathrm{R}} \mathrm{Cm}^{\mathrm{R}}$ & Altarriba et al. (2003) \\
\hline pACYC184-flgN & pACYC184 with complete $f g N$ gene, $\mathrm{Cm}^{\mathrm{R}}$ & This study \\
\hline
\end{tabular}

\section{General DNA methods}

Plasmid DNA was isolated using a plasmid DNA extraction kit (Omega Bio-Tek, Norcross, GA, USA). A. hydrophila chromosomal DNA isolation was carried out according to the instructions of the bacterial DNA kit (TaKaRa Bio, Otsu, Shiga, Japan). DNA restriction digestions and T4 ligations were carried out according to manufacturer's instructions. DNA samples were separated on 1\% agarose gels; when required, DNA extraction from gels was carried out using a Gel Extraction Kit (Omega Bio-Tek).

\section{Insertional mutagenesis of $A$. hydrophila}

The mini-Tn $10 \mathrm{Km}$ transposon was introduced into $A$. hydrophila on the suicide vector pLOFKm carried by E. coli Sm10 through a filter mating technique developed by Herrero et al. (1990) with minor modifications. Briefly, $0.22 \mu \mathrm{m}$ filters with a 1:4 mixture of the donor strain, E. coli Sm10 (pLOFKm) carrying the mini-Tn10Km, and the recipient strain, $A$. hydrophila, were incubated for $4 \mathrm{~h}$ at $28^{\circ} \mathrm{C}$ on LB plates supplemented with $3 \mathrm{mM}$ IPTG. The filters were transferred into Eppendorf tubes containing $1 \mathrm{~mL} \mathrm{LB}$ and vortexed. A $100-\mu \mathrm{L}$ suspension aliquot was spread onto LB plates supplemented with $600 \mu \mathrm{g}$ ampicillin and 100 $\mu \mathrm{g}$ streptomycin per $\mathrm{mL}$ to select for $A$. hydrophila carrying the mini- $\mathrm{Tn} 10 \mathrm{Km}$ transposon. Following incubation at $28^{\circ} \mathrm{C}$ for $24 \mathrm{~h}$, single colonies were selected for analysis in the adhesion assay. All colonies were further investigated with an indirect ELISA assay.

\section{Adhesion assay}

The adhesion assay was performed by the method of indirect ELISA using 96-well microtiter polystyrene plates, as previously described with some modifications (Collado et al., 2005). Briefly, $100 \mu \mathrm{L}$ sterilized eel skin mucus was immobilized into each well by incubation overnight at $4^{\circ} \mathrm{C}$. The negative control was sterile phosphate buffer saline (PBS). After incubation, the plates were washed twice with PBS to remove unbound mucus, and $100-\mu \mathrm{L}$ bacterial suspension was added into the wells and immobilized by incubation for $2 \mathrm{~h}$ before 
being washed three times by PBS plus Tween 20 and dried at $60^{\circ} \mathrm{C}$. The plates were blocked with $200-\mu \mathrm{L}$ fetal calf serum for $1 \mathrm{~h}$ at $37^{\circ} \mathrm{C}$ and washed in PBS-T. Subsequently, $100 \mu \mathrm{L}$ sera raised in rabbits against the antigen $A$. hydrophila was diluted to 1:100 with PBS and added to homologously antigen-coated wells, incubated at $37^{\circ} \mathrm{C}$ for $1 \mathrm{~h}$, and then washed three times in PBS-T. Rabbit anti-goat horseradish peroxidase (HRP) conjugate at a dilution of 1:1000 was added to each well and incubated at $37^{\circ} \mathrm{C}$ for $1 \mathrm{~h}$; the wells were then thoroughly washed and $50 \mu \mathrm{L}$ substrate solution [4 mg O-phenylenediamine tetrahydrochloride and $20 \mu \mathrm{L} \mathrm{H}_{2} \mathrm{O}_{2}$ $(30 \%, \mathrm{v} / \mathrm{v})$ in $10 \mathrm{~mL}$ acetate buffer, $\mathrm{pH} 5.0]$ was added. The plates were incubated at $37^{\circ} \mathrm{C}$ for $30 \mathrm{~min}$ in a dark chamber and finally the optical density (OD) was recorded at $492 \mathrm{~nm}$ using a microplate reader (Bio-Rad, Hercules, CA, USA).

\section{Molecular analysis of the mutant}

The DNA sequence flanking the transposon mutants was determined using thermal asymmetric interlaced polymerase chain reaction (TAIL-PCR). The arbitrary primer was supplied by the Genomic Walking Kit (TaKaRa Bio), and the nested primers specific to the transposon are listed in Table 2. Among the nested primers, LSP4-6 were used to amplify upstream sequences flanking the transposon, and RSP4-6 were used to amplify the downstream sequences flanking the transposon. TAIL-PCR products were purified using a Gel Extraction Kit (Omega Bio-Tek), after which they were cloned into pMD18-T (TaKaRa Bio) and sequenced. DNA sequences were analyzed by BLASTn (National Center for Biotechnology Information, Bethesda, MD, USA) and other softwares such as ClustalW and MegAlign (DNAStar).

\begin{tabular}{ll}
\multicolumn{2}{c}{ Table 2. Specific primers used in TAIL-PCR. } \\
\hline Primer & Sequence \\
\hline LSP4 & 5' ATGCTTGATGGTCGGAAGAGGC 3' \\
LSP5 & 5' CATCGGGCTTCCCATACAATCG 3' \\
LSP6 & 5' ATTATCGCGAGCCCATTTATACCC 3' \\
RSP4 & 5' CCTGTTGAACAAGTCTGGAAAGAAATG 3' \\
RSP5 & 5' GATCTTGCCATCCTATGGAACTG 3' \\
RSP6 & 5' TTACGCTGACTTGACGGGACGG 3' \\
\hline
\end{tabular}

\section{Construction of the complemented strain $(f g N+)$}

The mutant $f l g N$ gene was amplified using the primers NF: 5'-GGA TCC GAC CTC GTC CTC CGA CAA CC-3' and NR: 5'-GCA TGC CTT ACT AGA GGC TAG CAT AAT CAG GAA CAT CAT ACG GAA GGC CTT GAT GCC GAT GGA GC-3', where enzyme restriction sites are underlined, and the hemagglutinin (HA)-tag sequence is italicized. The PCR product was ligated to the pMD18-T vector (TaKaRa Bio) and introduced into E. coli DH5 $\alpha$. Single bacterial colonies were inoculated into $5 \mathrm{~mL} \mathrm{LB}$ medium containing $50 \mathrm{mg} / \mathrm{mL}$ ampicillin with shaking overnight at $37^{\circ} \mathrm{C}$. Recombinant plasmid was extracted and digested with BamHI and SphI. The digested products were subcloned into the expression vector pACYC184 previously digested with the same endonuclease, and the final construct was named pACYC184-flgN. Plasmid pACYC184-flgN was independently introduced into the mutant strain A77 by electroporation. Transconjugants were selected on chloromycetin plates. The expression of FlgN was detected by western blot according to supplier's instructions. 


\section{Western blot}

Whole extracts of the wild-type and complemented strains were resuspended in 0.5 $\mathrm{mL}$ loading buffer. Samples were heated for $10 \mathrm{~min}$ at $95^{\circ} \mathrm{C}$ and subjected to $12 \%$ SDS-polyacrylamide gel electrophoresis (PAGE). Proteins resolved by SDS-PAGE were transferred to polyvinylidene fluoride membranes (GE Healthcare, Little Chalfont, UK) and blocked overnight with PBS with $1 \%$ milk powder $(\mathrm{w} / \mathrm{v})$ at $4^{\circ} \mathrm{C}$ with gentle agitation. Blots were probed with the anti-HA tag (1:3000) and a goat HRP-conjugated secondary anti-mouse antibody (Sigma) (1:5000), and then developed using ECL Plus (Ruisai, Shanghai, China).

\section{Motility assay}

Freshly grown bacterial colonies were stabbed into the center of test tubes filled with semi-soft medium (LB base, $0.3 \%$ agar) and incubated at $28^{\circ} \mathrm{C}$ for $16-24 \mathrm{~h}$ to assess motility.

\section{Biofilm formation}

Quantitative biofilm formation was performed in a microtiter assay as described previously with minor modifications (Pratt and Kolter, 1998). Briefly, overnight cultures were diluted 1:100 into fresh broth. Then $100 \mu \mathrm{L}$ aliquots were added on a 96-well microtiter plate (polystyrene) and incubated in humidified conditions for $48 \mathrm{~h}$ at $30^{\circ} \mathrm{C}$ without shaking. To remove any unattached bacteria, the microtiter plates were rinsed thoroughly with sterile PBS and surface-attached bacteria were dried for $30 \mathrm{~min}$ at $60^{\circ} \mathrm{C}$, and subsequently stained with $1.0 \%$ crystal violet for $10 \mathrm{~min}$. After solubilization with $33 \%$ acetic acid, the OD was recorded at $590 \mathrm{~nm}$ using a microplate reader (Bio-Rad).

\section{Statistical analysis}

All data were statistically analyzed using analysis of variance tests in SPSS17.0 (SPSS, Chicago, IL, USA).

\section{RESULTS}

\section{Screening of adhesion-defective mutants}

A collection of 276 random insertion mutants of $A$. hydrophila was obtained using mini-Tn $10 \mathrm{Km}$ mutagenesis. Each mutant in the library was subjected to the adhesion assay to identify adhesion. The mutant strain with the most attenuated adhesion ability in this study was designated A77. The adhesion assay showed that the adhesion ability of A77 reduced approximately $50 \%$ compared to the wild-type strain (Figure 1).

\section{Sequence analysis of the $A$. hydrophila mini-Tn10 insertion site}

Sequence analysis showed that the 414-bp sequence flanking mini-Tn10 in A77 shared the highest identity (97\%) with a flagellar protein $f g N$ gene of $A$. hydrophila ATCC 7966 (accession No. CP000462.1) and with the FlgK/FlgL-specific chaperone $(f l g N)$ gene 
of A. hydrophila AL09-73 (accession No. Q216196.1). Analysis of the $f g N$ gene of the $A$. hydrophila wild-type strain showed that this gene contains a 414-bp open reading frame and encodes a FlgN protein which is predicted to have 137 amino acids of approximately $15 \mathrm{kDa}$ molecular weight.

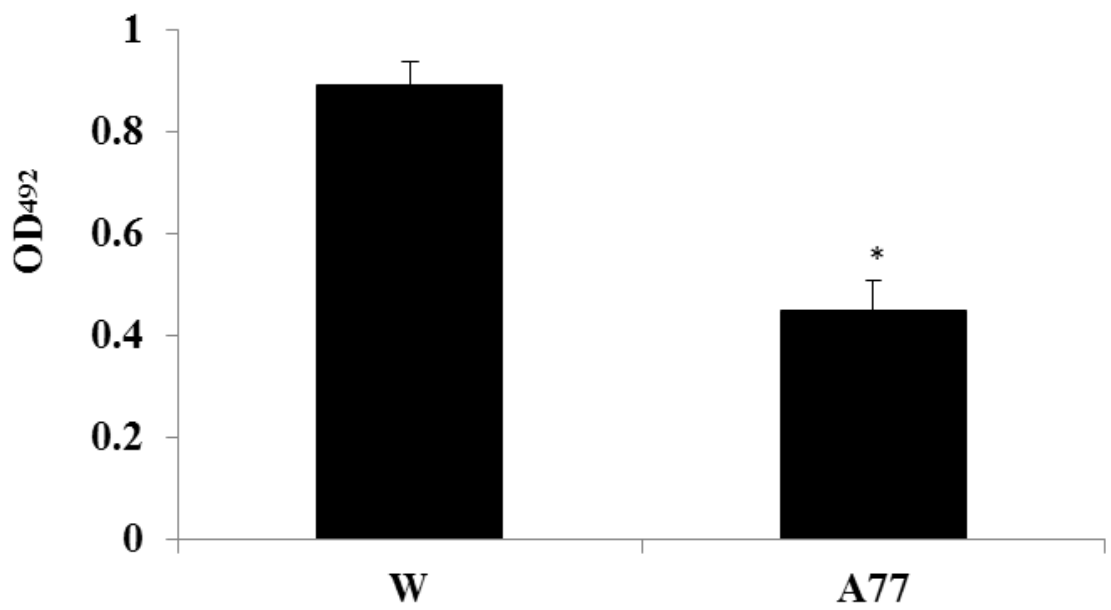

Figure 1. Adhesion of wild-type and mutant strain of Aeromonas hydrophila to fish mucus. Results are reported as the optical density (OD) recorded at $492 \mathrm{~nm}$ in a microplate reader (mean and SD). *Significant difference at $\mathrm{P}<$ 0.05 among adhesion difference from wild-type strain. All values are the average of three independent experiments.

\section{Western blot}

To detect the expression of the pACYC184-flgN vector in the complemented strain flgN+, western blot analysis was performed. The results demonstrated that the FlgN protein was detectable in the strain flgN+, which confirmed that the FlgN protein can be expressed in the complemented strain (Figure 2).

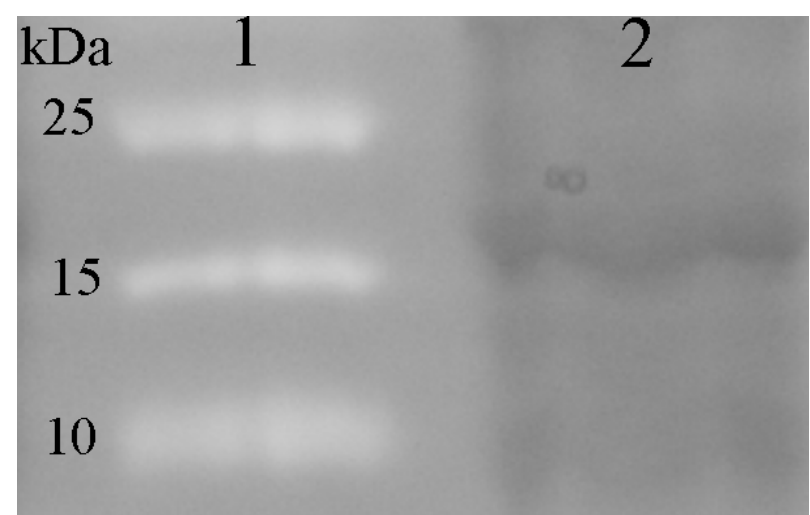

Figure 2. Western blot analysis of the complementation strain. Lane $1=$ protein markers; lane 2 = purified protein. 


\section{Bacterial motility}

The bacterial motility assay showed that all the strains migrated from the line of inoculation causing turbidity in the semi-soft medium. However, the mutant strain A77 displayed defective motility when compared with the wild type and complementation strains (Figure 3). This result suggests that mutation in $f g N$ can markedly attenuate bacterial motility.

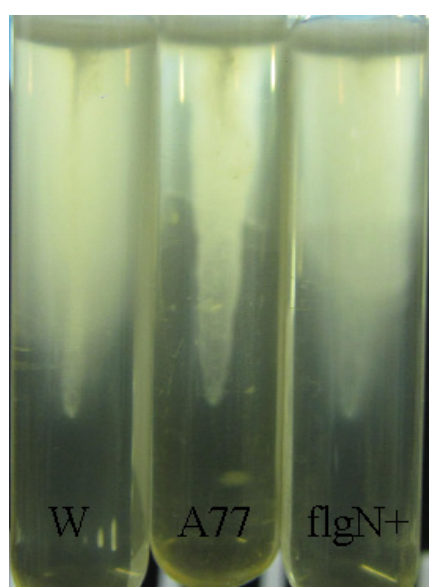

Figure 3. Motility of the wild-type, the mutant and the complementation in semi-soft medium.

\section{Adhesion assay}

The wild-type strain, the mutant strain A77, and the complemented strain flgN+ all demonstrated adhesion properties. The results showed that the number of adhesive bacteria of the mutant strain exhibited an approximate $75 \%$ reduction compared to the wild type strain, and that the numbers of adhesive bacteria of the complemented strain had recovered over $80 \%$ those of the wild-type strain (Figure 4). These data suggested that mutation in $f \lg N$ significantly affected bacterial adhesion.

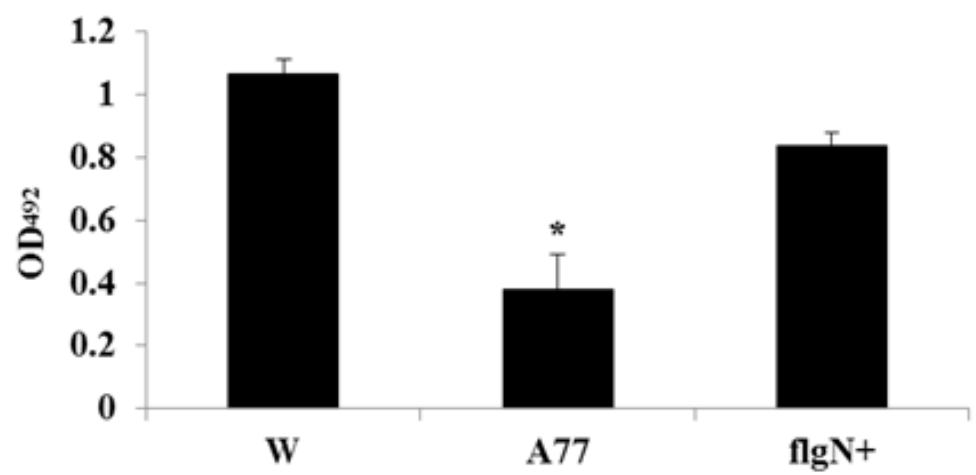

Figure 4. Adhesion of Aeromonas hydrophila wild-type, mutant and complementation strains to skin mucus of eel. Results are reported as the optical density (OD) recorded at $492 \mathrm{~nm}$ in a microplate reader (means and SD). *Significant difference from wild-type strain $(\mathrm{P}<0.05)$. All values are the average of three independent experiments. 


\section{Biofilm formation}

Solid surface-associated biofilm formation of these bacteria was also evaluated. The results revealed that the biofilm formed by the mutant strain was only about $35 \%$ of that formed by the wild-type strain as measured by optical density, while the biofilm formed by the complemented strain was restored to nearly $90 \%$ of that formed by the wild-type strain. These data suggested that mutation in flgN can attenuate bacterial biofilm formation (Figure 5).

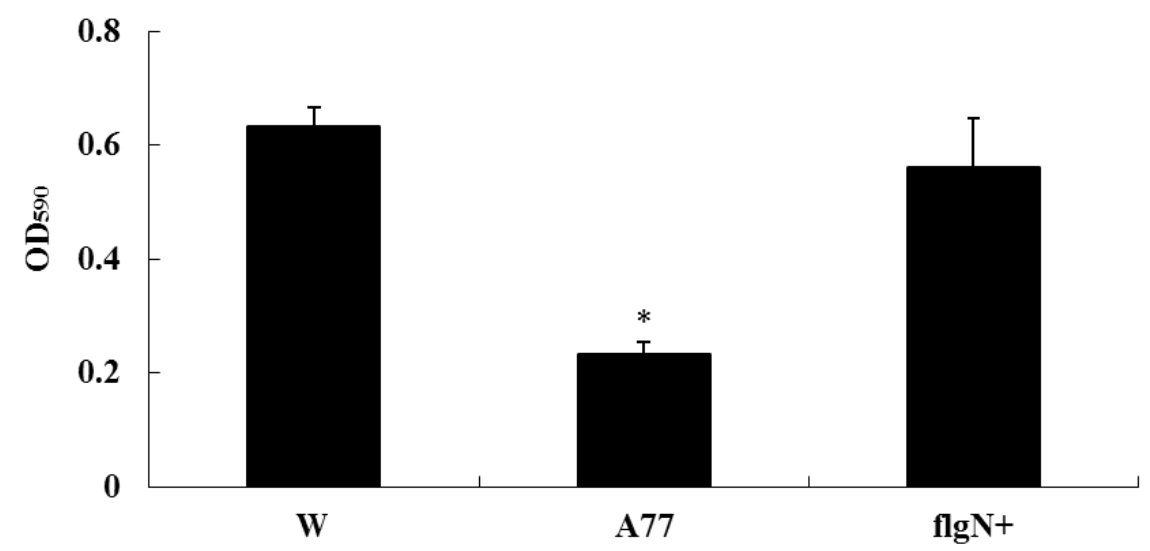

Figure 5. Biofilm formation of the Aeromonas hydrophila wild-type, mutant and complementation strains. $\mathrm{OD}_{590}$ quantifies the amount of crystal violet retained by the biofilm on the microtitre plates after staining. The average of three independent experiments (each experiment performed in duplicate) is shown. Error bars represent standard deviations. *Significant difference from wild-type strain $(\mathrm{P}<0.05)$.

\section{DISCUSSION}

Previous studies have shown that adhesion is one of the critical factors necessary for pathogenic bacteria to invade their host. In this study on A. hydrophila adhesion, we found that mutation in $f g N$ led to defective motility, adhesion, and biofilm formation.

It is known that $A$. hydrophila expresses two flagellar systems, a single polar flagellum for swimming in all culture conditions and multiple lateral flagella for swarming on solid or semisolid media (Gavín et al., 2002; Canals et al., 2006a,b). Studies on A. hydrophila flagellar gene systems has revealed that 55 genes are involved in the polar flagellum and 38 genes are involved in the lateral flagella (Merino et al., 2006), and that over 20 genes are shared by these two flagellar systems, which include the $f g N$ gene (Fraser et al., 1999). Further studies revealed that $\mathrm{FlgN}$ is an approximately $16-\mathrm{kDa}$ protein composed of 140 amino acid residues, and acts as a flagellar type III export chaperone specific for the hook-filament junction proteins, FlgK and FlgL (Yeh and Klesius, 2011). FlgN is also a translational regulator of the antisigma factor FlgM, and thus is predicted to play an important role in fine-tuning the flagellar assembly process (Minamino et al., 2012). Loss of FlgN reduces the initiation of filament assembly and the number of flagellar filaments, but not the stability of mature filaments, resulting in a motile but non-swarming mutant of Proteus mirabilis (Gygi et al., 1997). Disruption of the $f g N$ gene of Salmonella significantly abolished the motility of the pathogen (Minamino 
et al., 2012). These results suggest that FlgN plays different roles in the motility of different bacteria. In this study, FlgN mutation led to significantly defective motility in A. hydrophila, which suggested that FlgN plays an important role in its motility.

The data in our study also revealed that defective motility directly affected the adhesion and biofilm formation of $A$. hydrophila. This might be due to the close relationship between motility and adhesion and biofilm formation.

It is well known that there are different kinds of adhesins that exist on the bacterial surface, which permit the bacteria to recognize many different elements of host surfaces (Pizarro-Cerdá and Cossart, 2006). It is only when bacteria find the adaptors on the host surfaces that they can adhere to their host. Flagellar motility is necessary for bacteria adhesion and colonization, as has been confirmed by previous studies (Gavín et al., 2002). Rabaan et al. (2001) found that single mutations of $\mathrm{flaA}$ and $\mathrm{flaB}$ resulted in decreased adhesion of approximately $50 \%$, and that mutation of $f l a H$, flaJ, or both flagellin genes resulted in the complete loss of adhesion. Mutation in the $f g N$ gene of $A$. hydrophila AH-3 showed an approximately $80 \%$ reduction in adhesion to HEp- 2 cells compared to their wild type strain (Canals et al., 2006a). In our study, mutation in $f g N$ led to an approximately 50\% reduction in adhesion to host skin mucus. We interpreted this to mean that the bacteria utilize flagellar motility to search for the adaptors on host surfaces; if their motility is impaired, it becomes difficult for the bacteria to find the appropriate binding sites for adhesion.

It has also been well understood that the first step of biofilm formation is the initial adhesion to the solid surface (O'Toole et al., 2000). Therefore, flagellar motility and adhesion would also play an important role in the biofilm formation of many bacteria. Lemon et al. (2007) demonstrated that flagellum-mediated motility is critical for Listeria monocytogenes adhesion and biofilm formation. Mutation of $A$. hydrophila AH-3 FlgN also resulted in a $62 \%$ reduction in the ability to form biofilm compared with their wild type strains (Canals et al., 2006a). These data are similar to the result in our study that mutation in FlgN led to an approximately $65 \%$ reduction in bacterial biofilm formation.

Adhesion and biofilm formation are both involved in bacterial virulence, which has been demonstrated in Gardnerella vaginalis, Staphylococcus epidermidis, E. coli, and other bacteria (Cerca et al., 2005; Saldaña et al., 2009). Therefore, we believe that FlgN mutation might also attenuate the virulence of $A$. hydrophila through reduction of bacterial motility, adhesion, and biofilm formation.

\section{ACKNOWLEDGMENTS}

Research supported by grants from The National Natural Science Foundation of China under contract \#31272699, the Science and Technology Program of Xiamen under contract \#3502Z20133016, and the Science and Technology Program of Ocean \& Fisheries Bureau of Xiamen under contract \#201357. We gratefully acknowledge Prof. Nie and Dr. Y. Huang for providing some of the strains and plasmids used in this study.

\section{REFERENCES}

Acord J, Maskell J and Sefton A (2005). A rapid microplate method for quantifying inhibition of bacterial adhesion to eukaryotic cells. J. Microbiol. Methods 60: 55-62. 
Altarriba M, Merino S, Gavín R, Canals R, et al. (2003). A polar flagella operon (flg) of Aeromonas hydrophila contains genes required for lateral flagella expression. Microb. Pathog. 34: 249-259.

Canals R, Altarriba M, Vilches S, Horsburgh G, et al. (2006a). Analysis of the lateral flagellar gene system of Aeromonas hydrophila AH-3. J. Bacteriol. 188: 852-862.

Canals R, Ramirez S, Vilches S, Horsburgh G, et al. (2006b). Polar flagellum biogenesis in Aeromonas hydrophila. J. Bacteriol. 188: 542-555.

Cerca N, Pier GB, Vilanova M, Oliveira R, et al. (2005). Quantitative analysis of adhesion and biofilm formation on hydrophilic and hydrophobic surfaces of clinical isolates of Staphylococcus epidermidis. Res. Microbiol. 156: 506514.

Chhatwal GS (2002). Anchorless adhesins and invasins of Gram-positive bacteria: a new class of virulence factors. Trends Microbiol. 10: 205-208.

Collado MC, Gueimonde M, Sanz Y and Salminen S (2005). Adhesion of selected Bifidobacterium strains to human intestinal mucus and the role of adhesion in enteropathogen exclusion. J. Food Protect. 68: 2672-2678.

Daskalov H (2006). The importance of Aeromonas hydrophila in food safety. Food Control 17: 474-483.

Essex-Lopresti AE, Boddey JA, Thomas R, Smith MP, et al. (2005). A type IV pilin, PilA, contributes to adherence of Burkholderia pseudomallei and virulence in vivo. Infect. Immun. 73: 1260-1264.

Fraser GM, Bennett JC and Hughes C (1999). Substrate-specific binding of hook-associated proteins by FlgN and FliT, putative chaperones for flagellum assembly. Mol. Microbiol. 32: 569-580.

Gavín R, Rabaan AA, Merino S, Tomás JM, et al. (2002). Lateral flagella of Aeromonas species are essential for epithelial cell adherence and biofilm formation. Mol. Microbiol. 43: 383-397.

Gavín R, Merino S, Altarriba M, Canals R, et al. (2003). Lateral flagella are required for increased cell adherence, invasion and biofilm formation by Aeromonas spp. FEMS Microbiol. Lett. 224: 77-83.

Gygi D, Fraser G, Dufour A and Hughes C (1997). A motile but non-swarming mutant of Proteus mirabilis lacks FlgN, a facilitator of flagella filament assembly. Mol. Microbiol. 25: 597-604.

Hanahan D (1983). Studies on transformation of Escherichia coli with plasmids. J. Mol. Biol. 166: 557-580.

Herrero M, de Lorenzo V and Timmis KN (1990). Transposon vectors containing non-antibiotic resistance selection markers for cloning and stable chromosomal insertion of foreign genes in gram-negative bacteria. J. Bacteriol. 172: 6557-6567.

Kelly MT, MacCallum DM, Clancy SD, Odds FC, et al. (2004). The Candida albicans CaACE2 gene affects morphogenesis, adherence and virulence. Mol. Microbiol. 53: 969-983.

Khajanchi BK, Sha J, Kozlova EV, Erova TE, et al. (2009). N-acylhomoserine lactones involved in quorum sensing control the type VI secretion system, biofilm formation, protease production, and in vivo virulence in a clinical isolate of Aeromonas hydrophila. Microbiology 155: 3518-3531.

Khushiramani RM, Maiti B, Shekar M, Girisha SK, et al. (2012). Recombinant Aeromonas hydrophila outer membrane protein 48 (Omp48) induces a protective immune response against Aeromonas hydrophila and Edwardsiella tarda. Res. Microbiol. 163: 286-291.

Lemon KP, Higgins DE and Kolter R (2007). Flagellar motility is critical for Listeria monocytogenes biofilm formation. J. Bacteriol. 189: 4418-4424.

McCoy AJ, Koizumi Y, Toma C, Higa N, et al. (2010). Cytotoxins of the human pathogen Aeromonas hydrophila trigger, via the NLRP3 inflammasome, caspase-1 activation in macrophages. Eur. J. Immunol. 40: 2797-2803.

Merino S, Rubires X, Aguillar A, Guillot JF, et al. (1996). The role of the O-antigen lipopolysaccharide on the colonization in vivo of the germfree chicken gut by Aeromonas hydrophila serogroup O:34. Microb. Pathog. 20: 325-333.

Merino S, Shaw JG and Tomas JM (2006). Bacterial lateral flagella: an inducible flagella system. FEMS Microbiol. Lett. 263: 127-135.

Minamino T, Kinoshita M, Hara N, Takeuchi S, et al. (2012). Interaction of a bacterial flagellar chaperone FlgN with FlhA is required for efficient export of its cognate substrates. Mol. Microbiol. 83: 775-788.

O’Toole G, Kaplan HB and Kolter R (2000). Biofilm formation as microbial development. Annu. Rev. Microbiol. 54: 49-79.

Pianetti A, Battistelli M, Barbieri F, Bruscolini F, et al. (2012). Changes in adhesion ability of Aeromonas hydrophila during long exposure to salt stress conditions. J. Appl. Microbiol. 113: 974-982.

Pizarro-Cerdá J and Cossart P (2006). Bacterial adhesion and entry into host cells. Cell 124: 715-727.

Pratt LA and Kolter R (1998). Genetic analysis of Escherichia coli biofilm formation: roles of flagella, motility, chemotaxis and type I pili. Mol. Microbiol. 30: 285-293.

Rabaan AA, Gryllos I, Tomás JM and Shaw JG (2001). Motility and the polar flagellum are required for Aeromonas caviae adherence to HEp-2 cells. Infect. Immun. 69: 4257-4267. 
Saldaña Z, Xicohtencatl-Cortes J, Avelino F, Phillips AD, et al. (2009). Synergistic role of curli and cellulose in cell adherence and biofilm formation of attaching and effacing Escherichia coli and identification of Fis as a negative regulator of curli. Environ. Microbiol. 11: 992-1006.

Yeh H-Y and Klesius PH (2011). Over-expression, purification and immune responses to Aeromonas hydrophila AL09-73 flagellar proteins. Fish Shellfish Immunol. 31: 1278-1283. 